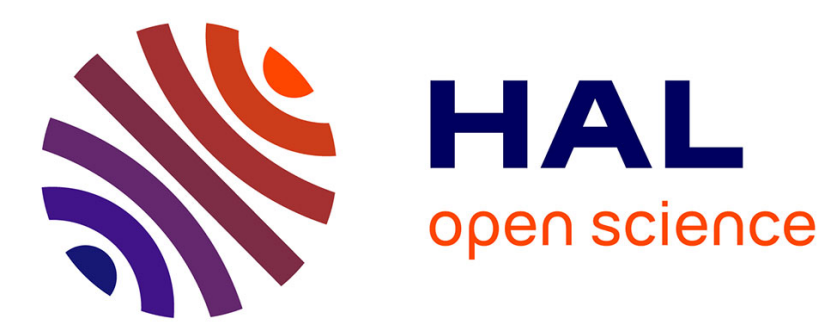

\title{
Anisotropic spectra of acoustic type turbulence
}

E. Kuznetsov, Vladimir Krasnoselskikh

\section{- To cite this version:}

E. Kuznetsov, Vladimir Krasnoselskikh. Anisotropic spectra of acoustic type turbulence. Physics of Plasmas, 2008, 15 (6), pp.062305. 10.1063/1.2928160 . insu-02928491

\section{HAL Id: insu-02928491 https://hal-insu.archives-ouvertes.fr/insu-02928491}

Submitted on 2 Sep 2020

HAL is a multi-disciplinary open access archive for the deposit and dissemination of scientific research documents, whether they are published or not. The documents may come from teaching and research institutions in France or abroad, or from public or private research centers.
L'archive ouverte pluridisciplinaire HAL, est destinée au dépôt et à la diffusion de documents scientifiques de niveau recherche, publiés ou non, émanant des établissements d'enseignement et de recherche français ou étrangers, des laboratoires publics ou privés. 


\section{Anisotropic spectra of acoustic type turbulence}

Cite as: Phys. Plasmas 15, 062305 (2008); https://doi.org/10.1063/1.2928160

Submitted: 10 April 2008 . Accepted: 15 April 2008 . Published Online: 10 June 2008

\section{E. Kuznetsov, and V. Krasnoselskikh}

\section{ARTICLES YOU MAY BE INTERESTED IN}

Whistler turbulence: Particle-in-cell simulations

Physics of Plasmas 15, 102305 (2008); https://doi.org/10.1063/1.2997339

Introduction to Focus Issue: Two-Dimensional Turbulence

Physics of Fluids 29, 110901 (2017); https://doi.org/10.1063/1.5012997

Experiments on rapidly rotating turbulent flows

Physics of Fluids 21, 096601 (2009); https://doi.org/10.1063/1.3197876

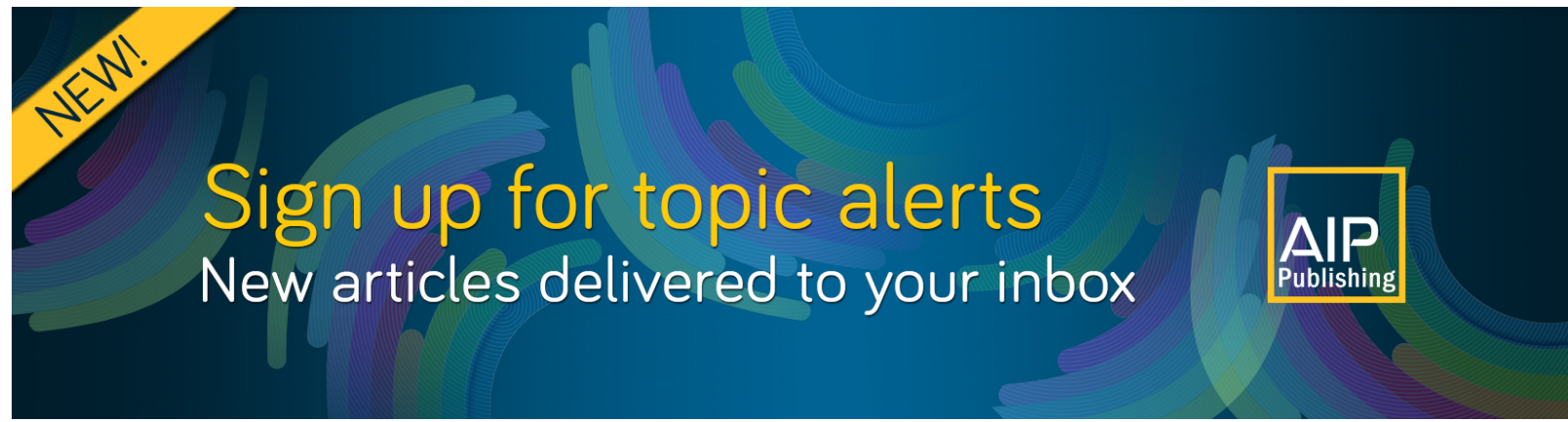




\title{
Anisotropic spectra of acoustic type turbulence
}

\author{
E. Kuznetsov ${ }^{1,2}$ and V. Krasnoselskikh ${ }^{1}$ \\ ${ }^{1}$ LPCE, 3 A Avenue de la Recherche Scientifique 45071 Orleans, CEDEX 2, France \\ ${ }^{2}$ P.N. Lebedev Physical Institute, 53 Leninsky Ave., 119991 Moscow, Russia
}

(Received 10 April 2008; accepted 15 April 2008; published online 10 June 2008)

\begin{abstract}
The problem of spectra for acoustic type of turbulence generated by shocks being randomly distributed in space is considered. It is shown that for turbulence with a weak anisotropy, such spectra have the same dependence in $k$-space as the Kadomtsev-Petviashvili spectrum: $E(k) \sim k^{-2}$. However, the frequency spectrum has always the falling $\sim \omega^{-2}$, independent of anisotropy. In the strong anisotropic case the energy distribution relative to wave vectors takes anisotropic dependence, forming in the large- $k$ region spectra of the jet type. (C) 2008 American Institute of Physics. [DOI: 10.1063/1.2928160]
\end{abstract}

\section{INTRODUCTION}

Acoustic type turbulence is ubiquitous in space and laboratory plasmas. Typical example represents magnetohydrodynamic (MHD) turbulence (for a review, see Ref. 1 and references therein) in the presence of external magnetic field at a moderate level of $\beta$ ( $\beta$ is the ratio of kinetic plasma pressure to the magnetic pressure). When wave amplitudes are small, the turbulence can be described as an ensemble of linear noninteracting waves with frequencies $\omega_{k}$ and wave vectors $k$. In the long-wave limit, the wave frequency can be considered as a linear function of wave number: $\omega_{k} \sim k$. In the thermodynamic equilibrium limit these waves are distributed according to the Jeans law so that the energy of each wave $\varepsilon_{k}=T$, where $T$ stands for the wave's temperature. When the wave amplitudes increase (with the growth of the energy source) we first come to the regime of weak turbulence. The nonlinear effects are weak in comparison with linear wave dispersion, but statistical characteristics of the system change significantly. In the weak linear turbulence regime, in addition to the thermodynamic distribution solution, it emerges the additional solution having the spectrum of the Kolmogorov type:

$$
\varepsilon(k)=C\left(\rho P c_{s}\right)^{1 / 2} k^{-7 / 2},
$$

where $P$ is the energy flux toward small scales, $\rho$ the density, $c_{s}$ the sound velocity, and $C$ is the dimensionless constant of the order of 1 . In the spherical normalization this energy distribution reads as follows:

$$
E(k)=4 \pi k^{2} \varepsilon_{k}=4 \pi C\left(\rho P c_{s}\right)^{1 / 2} k^{-3 / 2} .
$$

This spectrum was found in 1971 by Zakharov and Sagdeev ${ }^{2}$ as the exact solution of the wave kinetic equation for acoustic waves in isotropic medium, when in the long-wave region, the dispersion relation is linear; i.e., $\omega_{k} \approx c_{s} k$ (for details, see Ref. 3). In this case, the criterion of weak turbulence, which is determined as the weakness of the nonlinearity with respect to the wave dispersion, is written as

$$
\frac{\Delta \rho}{\rho} \ll k^{2} \Lambda^{2},
$$

where $\Delta \rho$ is the fluctuating part of the density $\rho$, and $\Lambda$ is the dispersion length. ${ }^{5}$ For larger amplitudes when the nonlinear effects become comparable or larger, the wave dispersion this criterion (2) breaks down. As is well known in gas dynamics, in this case the main nonlinear effect for acoustic waves is nothing else but the wave breaking that results in the formation of shocks (or discontinuities). It is also well known that this process in compressible flows can be treated in terms of the formation of folds in the classical catastrophe theory (see, for example, Ref. 6). In the gas-dynamic case, breaking areas can be completely characterized using the mapping defined by the transition from the usual Eulerian to the Lagrangian description. A vanishing of the Jacobian of the mapping corresponds to the emergence of a singularity for the spatial derivatives of the velocity and density of the fluid. The physical meaning of this effect corresponds to the intersection of particle trajectories. In the general situation, the first time the Jacobian vanishing happens in one isolated point. In collisionless plasma this process can continue and leads further to form the multiflow region expanding in the transverse (relative to the main flow) direction according to the following scaling law: $R_{\perp} \sim \sqrt{t_{0}-t}$, where $t_{0}$ stands for the first moment when Jacobian $J$ turns to zero, while the region width (in the longitudinal direction, along the main flow) increases more slowly; i.e., $R_{\|} \sim\left(t_{0}-t\right)^{3 / 2}$ (see, e.g., Refs. 7 and 8). Thus, the result of the breaking consists in the formation of structures in the form of pancakes with very different characteristic spatial scales along the flow and in transverse directions. In optics, quite similar structures are called caustics (see, for example, Ref. 9). The simplest way to represent such structures is to consider them as disks (singular manifolds) on which the density undergoes jumps vanishing at the disk boundary. It is worth noting that in classical hydrodynamics these regions are considered as "forbidden," where the solution can no longer be constructed in a well defined way, which means as a single value solution. There are special procedures of construction of so-called "shock type" solution (see the book of Whitham ${ }^{10}$ for more detail). However, in collisionless plasma physics, the detailed de- 
scription is written in terms of velocity distribution function that satisfies the Vlasov equation in the phase space $\left(\mathbf{p}_{\mathbf{i}}, \mathbf{r}_{\mathbf{i}}\right)$. For this function, the regions where the hydrodynamic solution becomes multivalued and poorly determined are the areas where the distribution function undergoes the transition from the one single peak distribution to the one having three peaks; however, there is no any crucial break in the phase space. On the other hand, the spatial derivatives of some characteristics have infinite gradients also, but this effect can be explicitly described in the frame of the same Vlasov equation for collisionless plasmas. Formation of simple waves making use of such a description was considered by Gurevich and Pitaevskii. ${ }^{11}$

In weak turbulence, waves are assumed to be randomly distributed with a weak correlation between the waves because of weak nonlinear interaction. The process of breaking is purely coherent and, respectively, the expanding caustics should be treated as coherent elementary entities of wave turbulence. We will call the turbulence where this effect becomes important a moderately strong turbulence if the density of caustics is still small enough to neglect their intersections that are supposed to be rare. The caustics can be described if their centers and orientations are determined. We will assume their distribution in space to be random. On the other hand, the jumps as the density singularities are known to result in a power-law tails in the short-wavelength part of the turbulence spectrum. This idea was first proposed by Phillips ${ }^{12}$ and allowed him to determine the water-wave turbulence spectrum in the presence of whitecaps; i.e., of the singularities on the fluid surface. Later, the very same idea was developed by Saffman ${ }^{13}$ to determine the isotropic spectrum of two-dimensional hydrodynamic turbulence at high Reynolds numbers (for details, see Refs. 14 and 15). Kadomtsev and Petviashvili (KP) as given in Ref. 16 suggested that the acoustic turbulence can be considered as a randomly distributed set of shocks. For the isotropic case they found the spectrum of the energy distribution, now known as the KP spectrum,

$$
E(k) \sim k^{-2} .
$$

The goal of this paper is to determine how the KP spectrum is modified in the presence of strong enough anisotropy in plasma. Such an anisotropy can be entirely inherent, either due to the mean magnetic field or because of the anisotropy of turbulence source/pumping. It is worth noting that in the weak-turbulence regime, when the wave dispersion of acoustic waves can be neglected, the angular distribution of the spectrum repeats the anisotropy of the pumping because of three-wave resonant conditions; i.e., $\omega_{k}=\omega_{k_{1}}+\omega_{k_{2}}$ and $\mathbf{k}$ $=\mathbf{k}_{1}+\mathbf{k}_{2}$. In this case the additional anisotropy of the spectra can appear either due to weak dispersion (see Refs. 3 and 17) or because of nonlinear renormalizations to the wave kinetic equation as given in Ref. 18. For weak MHD turbulence, in both low and high beta plasmas, the situation is familiar to the weak acoustic turbulence in isotropic media for the nonlinear interacting fast magnetoacoustic waves, the dispersion of which is defined mainly by the modulus of the wave vector. However, at $\beta \ll 1$, slow magnetoacoustic waves undergo strong anisotropy in both dispersion and nonlinear interac- tion due to the external magnetic field. As was shown in a previous paper ${ }^{19}$ by one of the authors, it results in the appearance of anisotropic Kolmogorov-type spectra with power dependences relative to both longitudinal and transverse projections of the wave vector. Such character of the spectra can be established also for the interaction of Alfvénic and slow magnetoacoustic waves at low beta plasma as given in Ref. 4. For $\beta \gg 1$, slow magnetoacoustic waves have the same dispersion as the Alfvénic waves. This degeneracy also changes significantly the nonlinear interaction between waves and leads in the weak-turbulence regime to the spectrum of the Kraichnan-Iroshnikov type ${ }^{20}$ (for details, see Refs. 1 and 21).

In the present paper, for moderately strong acoustic turbulence, we study how the angular ordering of shocks can change the angular structure of the spectrum. We show that for the strong anisotropy the spectrum has the jet-type behavior with power increasing along the jet with the same exponent as for the isotropic KP spectrum and the falling-off dependence in the transverse direction: $\sim k_{\perp}^{-5}$. The latter originates from the contribution from boundaries of caustics (disks). It is necessary to mention that for two-dimensional acoustic turbulence the phenomenon of the jet-type spectra generated by shocks was observed for the first time in the numerical experiments as given in Ref. 22. However, comparably small spatial resolution could not allow the authors of Ref. 22 to treat the fine structure of jets.

The second objective of this work is to show how the presence of the KP spectrum and its relative role can be evaluated making use of real experimental data (for instance, from spacecraft data for solar wind turbulence) in the presence of weak-turbulence tails falling off more slowly than the KP spectrum. This question is very important because in the case when weak and strong turbulent components coexist, the KP component of the spectrum can provide a quantitative measure of the relative role of coherent structures for moderate acoustic type turbulence. Hereafter, we shall also show that the spectral index of the isotropic KP spectrum [Eq. (3)] as a function of the wave vector does not depend on space dimension. It is worth noting that the Fourier transform of the density single point autocorrelation function dependence upon time shows the same power-law spectral index in time domain (in frequency) as in the spatial domain (spectrum in $k$ ) [Eq. (3)].

\section{ISOTROPIC SPECTRA}

Let us consider the contribution of shocks to the frequency spectrum; i.e., the energy distribution dependence on frequency. To find the spectrum one should calculate the autocorrelation function for the density $\rho(t)$ measured at some point $\mathbf{r}_{0}$ as a function of time, i.e., $K(\tau)=\langle\rho(t+\tau) \rho(t)\rangle$, where the angular brackets stand for time averaging of the (mass) density $\rho(t)$, and then carry out the Fourier transform $K(\tau)$ :

$$
K_{\omega}=\int_{-\infty}^{\infty} K(\tau) e^{i \omega \tau} d \tau
$$

Here the density distribution is assumed to be homogeneous, so that $K_{\omega}$ does not depend on $\mathbf{r}_{0}$. It is worth noting that 
defined in such a way, $K_{\omega}$ coincides, up to constant factor, with the energy spectrum in the frequency domain:

$$
E_{\omega}=\frac{c_{s}^{2}}{\rho_{0}} K_{\omega} .
$$

In the weak-turbulence approximation, in an isotropic case $E_{\omega}$ can be expressed in terms of the spectrum $\varepsilon(k)$ by means of the relation

$$
E_{\omega}=\frac{4 \pi \omega^{2}}{c_{s}^{3}} \varepsilon(k),
$$

where $k=\omega / c_{s}$. This formula is the result of integration of the energy spectral density in the $k-\omega$ representation $E_{k \omega}$ $=\varepsilon(k) \delta\left(\omega-\omega_{k}\right)$ with respect to $\mathbf{k}$. In the weak-turbulence regime, the spectral density $E_{k \omega}$ has the $\delta$-function dependence upon frequency indicating that the wave ensemble is weakly nonlinear. In the presence of shocks, i.e., for the strongturbulence regime, such relations are no longer valid.

The aim now is to determine the contribution of shocks associated with the density jumps to the $E_{\omega}$ spectrum. To achieve this one should take into account that at the instant $t_{i}$ of jump passage through the measurement point $\mathbf{r}_{0}$, the first derivative $\partial \rho / \partial t$ is proportional to $\delta\left(t-t_{i}\right)$; i.e.,

$$
\frac{\partial \rho}{\partial t}=\sum_{i} \Delta \rho_{i} \delta\left(t-t_{i}\right)+\text { regular terms } .
$$

Assuming that density jumps $\Delta \rho_{i}$ and crossing times $t_{i}$ are random quantities one can calculate the contribution of these singularities in Eq. (5) to the spectrum. The Fourier transform of the contribution associated with these terms can be written as follows:

$$
\rho_{\omega}=\frac{i}{2 \pi \omega} \sum_{i} \Delta \rho_{i} e^{-i \omega t_{i}}
$$

Here,

$$
\rho_{\omega}=\int_{-\infty}^{\infty} \rho(t) e^{i \omega t} d t, \quad \rho(t)=\frac{1}{2 \pi} \int_{-\infty}^{\infty} e^{-i \omega t} \rho_{\omega} d \omega .
$$

To find the $E_{\omega}$ spectrum, one should square the absolute value of Eq. (6) and average the resulting expression. The averaging over crossing times $t_{i}$ yields

$$
E_{\omega}=\frac{c_{s}^{2}}{2 \pi \rho_{0} \tau}\left\langle\left|\rho_{\omega}\right|^{2}\right\rangle=\frac{c_{s}^{2} \nu}{2 \pi \rho_{0} \omega^{2}} \overline{(\Delta \rho)^{2}},
$$

where $\nu=N / \tau$ is the jump crossings frequency, here $N$ is the number of discontinuities met during the averaging time $\tau$, and $\overline{(\Delta \rho)^{2}}$ is the average value of $(\Delta \rho)^{2}$.

The very same approach can be applied for finding the spatial spectrum of acoustic turbulence for the onedimensional case $(D=1)$, when instead of Eq. (5), we have

$$
\frac{\partial \rho}{\partial x}=\sum_{i} \Delta \rho_{i} \delta\left(x-x_{i}\right)+\text { regular terms }
$$

where $x_{i}$ are the positions of jumps along the $x$ axis. Hence, we obtain the following one-dimensional spectrum $E(k)$ :

$$
E_{1}(k)=\frac{n_{1} c_{s}^{2}}{2 \pi \rho_{0} k^{2}} \overline{(\Delta \rho)^{2}} .
$$

Here, $n_{1}$ is the number density of shocks per unit length, $\rho_{0}$ is the mean density of medium (per unit length), and $\overline{(\Delta \rho)^{2}}$ is the mean-square of the density jump at the discontinuity.

This calculation can be considered in isotropic threedimensional (3D) case as the estimate of the correlation function along any chosen straight line. Now we shall make use of it to obtain the 3D isotropic spectrum [Eq. (9)]. To this end, one should notice that the density correlation function for isotropic turbulence, i.e., $\phi\left(y_{1}\right)=\left\langle\rho\left(x_{1}\right.\right.$ $\left.\left.+y_{1}, x_{2}, x_{3}\right) \rho\left(x_{1}, x_{2}, x_{3}\right)\right\rangle$, has a Fourier spectrum (with respect to only one single variable $y_{1}$ !) that coincides, to within a factor, with Eq. (9):

$$
\phi_{k}=\frac{N_{1}}{2 \pi k^{2}} \overline{(\Delta \rho)^{2}}
$$

where $N_{1}$ in this case should be considered as the mean linear density of discontinuities. This correlation function $\phi_{k}$ is related to the three-dimensional Fourier spectrum

$$
\Phi(|\mathbf{k}|)=\int \phi(\mathbf{r}) e^{-i \mathbf{k} \cdot \mathbf{r}} d \mathbf{r}
$$

by the following formula:

$$
\phi_{k_{1}}=\int \Phi(|\mathbf{k}|) d \mathbf{k}_{\perp}=\pi \int_{k_{1}^{2}}^{\infty} \Phi(s) d s^{2},
$$

where $\mathbf{k}_{\perp}$ is the component of the wave vector $\mathbf{k}$ perpendicular to the $x$ axis. This allows one to obtain, differentiating this equality with respect to $k_{1}$, the following relation:

$$
\Phi(k)=-\frac{1}{2 \pi k} \frac{d \phi_{k}}{d k} .
$$

Substituting Eq. (10), one can then find the following expression for the spectrum $E_{3}(k)$ :

$$
E_{3}(k)=\frac{2 N_{1} c_{s}^{2}}{\pi \rho_{0} k^{2}} \overline{\Delta \rho^{2}} .
$$

The same approach is applicable in the two-dimensional case. It is a little more difficult technically because it requires to solve the integral Abel equation (e.g., compare with Refs. 13 and 14). However, making calculations one can find that the spectrum dependence upon $k$ has the same spectral index as in one- and three-dimensional cases:

$$
E_{2}(k) \propto k^{-2} .
$$

According to the knowledge of the authors' spectrum [Eq. (9)] was first obtained by Burgers, as it was mentioned by Saffman in his paper, ${ }^{13}$ and, its generalization for multidimensional situation was found [Eq. (11)] by Kadomtsev and Petviashvili, as given in Ref. 16. In the next section we show how to generalize it taking into account the effect of anisotropy. 


\section{ANISOTROPIC KP SPECTRA}

The analysis of anisotropic situation consists in taking account of two geometric factors. One is connected with the anisotropy of the caustics' orientations, another with the anisotropy due to the "emission" diagram of each caustic that can be considered as the source of the wave spectrum. For the sake of simplicity and without loss of generality, we can consider that each single caustic has the form of a disk. This simplification is based on important property of these objects; namely, during their evolution, most of the time their relative size in the perpendicular direction is much larger than along the parallel axis.

Let us consider a single caustic with radius $R_{i}$ perpendicular to the $x$ axis and centered at the point $\mathbf{r}_{0}=\left(x_{0}, \mathbf{r}_{\perp 0}\right)$. To consider this effect, Eq. (8) should be replaced by the following one:

$$
\frac{\partial \rho}{\partial x}=\Delta \rho\left(\left|\mathbf{r}_{\perp}-\mathbf{r}_{\perp 0}\right|\right) \delta\left(x-x_{0}\right)+\text { regular terms } .
$$

Here, $\Delta \rho\left(r_{\perp}\right)$ is considered as a continuous, cylindrically symmetric function of $r_{\perp}$ that vanishes at the disk boundary, i.e., $r_{\perp}=R[\Delta \rho(R)=0]$, and remains zero outside the disk.

The Fourier transform of the terms corresponding to singular part of Eq. (12) is then given by the integral

$$
\begin{aligned}
\rho_{\mathbf{k}} & =-\frac{i}{k_{x}} e^{-i \mathbf{k} \mathbf{r}_{0}} \int_{r_{\perp} \leqslant R} \Delta \rho\left(r_{\perp}\right) e^{-i \mathbf{k}_{\perp} \mathbf{r}_{\perp}} d \mathbf{r}_{\perp} \\
& =-\frac{2 \pi i}{k_{x}} e^{-i \mathbf{k r}_{0}} \int_{0}^{R} r_{\perp} \Delta \rho\left(r_{\perp}\right) J_{0}\left(k_{\perp} r_{\perp}\right) d r_{\perp},
\end{aligned}
$$

where $\mathbf{k}=\left(k_{x}, \mathbf{k}_{\perp}\right)$ and $J_{0}\left(k_{\perp} r_{\perp}\right)$ is the Bessel function. This is the contribution from one single singularity. The total contribution from all discontinuities can be found as the sum:

$$
\rho_{\mathbf{k}}=-2 \pi i \sum_{\alpha} \frac{e^{-i \mathbf{k} r_{\alpha}}}{\mathbf{k n}_{\alpha}} \int_{0}^{R_{\alpha}} \Delta \rho\left(r_{\perp}\right) r_{\perp} J_{0}\left(k_{\perp \alpha} r_{\perp}\right) d r_{\perp} .
$$

Here, $\mathbf{n}_{\alpha}$ is the normal unit vector to the discontinuity $\alpha, \mathbf{r}_{\alpha}$ are the disk center coordinates and $k_{\perp \alpha}$ is the transverse projection of the wave vector $\mathbf{k}$ to the disk plane $\left[k_{\perp \alpha}^{2}=k^{2}\right.$ $\left.\left.-(\mathbf{k n})_{\alpha}\right)^{2}\right]$. It is worth noting here that the anisotropic characteristics of the spectrum is related to the anisotropy of the distribution of unit vectors $\mathbf{n}_{\alpha}$.

To find the spectrum of turbulence, one should average $\left|\rho_{k}\right|^{2}$ over all random variables. It is natural to assume that the coordinates $\mathbf{r}_{\alpha}$ of centers of caustics are distributed uniformly; the averaging over these variables results in

$$
\overline{\left|\rho_{k}\right|^{2}}=4 \pi^{2} N\left\langle\left|\frac{1}{k_{\|}} \int_{0}^{R} \Delta \rho\left(r_{\perp}\right) r_{\perp} J_{0}\left(k_{\perp} r_{\perp}\right) d r_{\perp}\right|^{2}\right\rangle .
$$

Here, $k_{\|} \equiv \mathbf{k n}, N$ is the density of discontinuities, and the angular brackets stand for the averaging over characteristic sizes $R$ and angles.

The major interest here is in evaluation of the shortwavelength asymptotic behavior of the spectrum found [Eq. (13)], i.e., $k \bar{R} \gg 1$, where $\bar{R}$ is the characteristic disk radius. Thus, if $k_{\perp} \bar{R} \gg 1$, then the expression inside the integral in
Eq. (13) represents rapidly oscillating function [due to the Bessel function $J_{0}\left(k_{\perp} r_{\perp}\right)$ ], and therefore the integral can be evaluated by means of the stationary phase method. Using the relation

$$
x J_{0}(\alpha x)=\frac{1}{\alpha} \frac{d}{d x}\left[x J_{1}(\alpha x)\right],
$$

and integrating by parts, we have

$$
\begin{aligned}
\int_{0}^{R} & \Delta \rho\left(r_{\perp}\right) r_{\perp} J_{0}\left(k_{\perp} r_{\perp}\right) d r_{\perp} \\
& =-\frac{R}{k_{\perp}} \int_{0}^{1} J_{1}\left(k_{\perp} R x\right) x \frac{d}{d x} \Delta \rho(R x) d x,
\end{aligned}
$$

where the property $\Delta \rho(R)=0$ is used. Having in mind that the major input to the integral comes from the vicinity of the boundary at $r_{\perp}=R$, we can use the asymptotic expression for the Bessel function at large $k_{\perp} R$,

$$
J_{1}(z) \simeq \sqrt{\frac{2}{\pi z}} \cos \left(z-\frac{3 \pi}{4}\right),
$$

and obtain the following asymptotic estimate of the integral,

$$
\begin{array}{r}
-\sqrt{\frac{2 R}{\pi}} \frac{1}{k_{\perp}^{3 / 2}} \int_{0}^{1} \cos \left(k_{\perp} R x\right) x^{1 / 2} \frac{d}{d x} \Delta \rho(R x) d x \\
\sim-\left.\sqrt{\frac{2 R}{\pi}} \frac{\sin \left(k_{\perp} R\right)}{k_{\perp}^{5 / 2}} \frac{d}{d r_{\perp}} \Delta \rho\left(r_{\perp}\right)\right|_{r_{\perp}=R},
\end{array}
$$

where the main contribution to the integral comes explicitly from the boundary $r_{\perp}=R$.

Hence, the resulting spectrum $\widetilde{\boldsymbol{\epsilon}}(\mathbf{k})$ (still prior to angular averaging!) can be written as follows:

$\tilde{\epsilon}_{1}(\mathbf{k})=\frac{4 \pi n c_{s}^{2}}{\rho_{0}} \frac{\langle\Psi\rangle}{k_{\perp}^{5} k_{\|}^{2}}, \quad \Psi=R\left(\left.\frac{d}{d r_{\perp}} \Delta \rho\left(r_{\perp}\right)\right|_{r_{\perp}=R}\right)^{2}$.

It is worth noting that the stationary phase method used for the evaluation of the integral in Eq. (13) is applicable at almost all angles $\theta$ ( $\theta$ is the angle between the vectors $\mathbf{k}$ and n), but not in two narrow cones; i.e., $\theta \leqslant \vartheta_{0}=(k \bar{R})^{-1}$ and $\pi$ $-\theta \leqslant \vartheta_{0}$. Inside these cones one can find that the integral can be considered to be independent of $k$ [suggesting $\left.\cos \left(k_{\perp} R x\right) \approx 1\right]$. The spectrum $\widetilde{\boldsymbol{\epsilon}}(\mathbf{k})$ inside these cones is then given by

$$
\widetilde{\epsilon}_{2}(\mathbf{k}) \approx \frac{4 \pi n c_{s}^{2}}{\rho_{0}} \frac{\left\langle\Gamma^{2}\right\rangle}{k_{\|}^{2}},
$$

where

$$
\Gamma=\int_{0}^{R} \Delta \rho\left(r_{\perp}\right) r_{\perp} d r_{\perp} .
$$

From Eq. (14) one can see that the spectrum $\widetilde{\epsilon}_{1}(\mathbf{k})$ contains three singularities; namely, at angles $\theta$ close to $0, \pi$, and $\pi / 2$. For angles close to the cone, i.e., $\theta \approx(k \bar{R})^{-1}$ and $\pi-\theta$ $\approx(k \bar{R})^{-1}$, expression (14) matches Eq. (15). For angles close to $\pi / 2$, in Eq. (14), an additional effect should be taken into 
account; namely, the bending of the caustics. If $a$ is the characteristic value of bending, Eq. (14) is valid in the region $|\theta-\pi / 2|>(k a)^{-1}$.

Distributions (14) and (15) allow one to carry out the averaging over angular distribution of caustics normals, if their distribution is anisotropic; i.e., calculate the angular dependence of the energy spectrum $E(\mathbf{k})=k^{2} \overline{\widetilde{\epsilon}(\mathbf{k})}$ in short enough scales: $(k \bar{R}) \gg 1$.

First, let us check that in the isotropic case, the KP spectrum [Eq. (11)] follows from the above calculations. In this case, averaging over the angles corresponds to the integration of Eqs. (14) and (15) with respect to $\theta$. The integration of expression (15) near the poles $(\theta=0, \pi)$ gives

$$
E_{2}(k)=4 \pi k^{2} \int_{0}^{\theta_{0}} \tilde{\epsilon}_{2}(k) \theta d \theta=\frac{16 \pi^{2} n c_{s}^{2}}{\rho_{0} k^{2}} \frac{\left\langle\Gamma^{2}\right\rangle}{\bar{R}^{2}} .
$$

In the integration of Eq. (14) over angles, the main contribution to the spectrum comes from the angles close to $0, \pi$ and $\pi / 2$, where the spectrum (14) has singularities. For $\theta \rightarrow 0$ (for $\theta \rightarrow \pi$ ), the integration is cut off at angles $\theta_{k}=\vartheta_{0}$ (at $\left.\theta_{k}=\pi-\vartheta_{0}\right)$, and for $\theta \rightarrow \pi / 2$, it is cut off at angles $\left|\pi / 2 \pm \theta_{k}\right| \approx(k a)^{-1}$. As the result of averaging of the expression (14), we obtain the main contributions coming from the angles near the cone:

$$
E_{1}(k) \approx \frac{16 \pi^{2} n c_{s}^{2}}{\rho_{0} k^{2}} \frac{\bar{R}^{3}\langle\Psi\rangle}{3} .
$$

The spectrum $E(k)$ is given by the sum of Eqs. (16) and (17),

$$
E(k) \approx \frac{16 \pi^{2} n c_{s}^{2}}{\rho_{0} k^{2}}\left(\frac{\left\langle\Gamma^{2}\right\rangle}{\bar{R}^{2}}+\frac{\bar{R}^{3}\langle\Psi\rangle}{3}\right),
$$

which has the same dependence on $k$ as the KP spectrum (11) obtained in a little different way from conventional consideration.

The above-performed analysis of spectral angular dependence upon the angle that is applicable first of all to one single caustic leads to the conclusion that the "emission diagram" of it represents narrow cones around $\theta=0, \pi / 2, \pi$. The angular width of these cones is proportional to $(k \bar{R})^{-1}$ around $\theta=0, \pi$ and to $(k a)^{-1}$ around $\theta=\pi / 2$. In the case of anisotropic distribution of caustics normals, the angular dependence of the spectrum will simply reproduce this angular dependence if the width $\Delta \theta$ of this angular distribution is sufficiently larger than the width of these cones. This can be summarized as follows. If the angular distribution of caustics normals is wide enough so that $\Delta \theta>\vartheta_{0}$, then after averaging of $\widetilde{\boldsymbol{\epsilon}}(\mathbf{k})$, one should get the same dependence of the spectrum upon $k$ at large enough $k$ as for the isotropic KP spectrum, i.e., $\sim k^{-2}$, with the degree of the anisotropy exactly the same as the distribution of caustics normals. On the other hand, if the angular distribution of caustics normals is sufficiently narrow, i.e., if all falling shock fronts are oriented almost unidirectionally (this can be caused, e.g., by pumping or initial/boundary conditions), then the spectrum will have a sharp peak in this direction. If the width $\Delta \theta$ of the angular distribution is narrower than $\vartheta_{0}$, i.e., if $\Delta \theta<\vartheta_{0}$, then the spectrum $E(k, \theta)$ up to the multiplier $k^{2}$ will repeat the distribution given by Eqs. (14) and (15); namely, the distribution in $k$-space will have the form of the jet. In the cone $\theta$ $<\vartheta_{0}$, the spectrum has a maximum with a falloff at large $k$ in accordance with Eq. (14), i.e., $\sim k_{\|}^{-2}$, like the KP spectrum (11). At larger angles, i.e., $\theta>\vartheta_{0}$, the spectrum $E(k, \theta)$ will rapidly decrease in the transverse direction proportionally to $k_{\perp}^{-5}$.

Note, however, that this asymptotic behavior is intermediate, because $\vartheta_{0}=(k \bar{R})^{-1}$ decreases with increasing $k$. For this reason, when averaging over angles, singularities in Eq. (14) become essential for $\theta \rightarrow 0$, and, starting from certain $k=k^{*}$, the spectrum will decrease as $k^{-2}$ with increasing $k$. The angular width of the spectrum will be of order $\Delta \theta$.

\section{ACKNOWLEDGMENTS}

The work of E.K. was partially supported by the Russian Foundation for Basic Research (Grant No. 06-01-00665), by the Council for the State Support of the Leading Scientific Schools of Russia (Grant No. NSH-4887.2008.2), and by a Poste Rouge fellowship of the French National Centre of Scientific Research.

${ }^{1}$ A. A. Schekochihin and S. C. Cowley, In: Magnetohydrodynamics: Historical Evolution and Trends, edited by S. Molokov, R. Moreau, and H. K. Moffatt (Springer, Berlin, 2007), p. 85.

${ }^{2}$ V. E. Zakharov and R. Z. Sagdeev, Sov. Phys. Dokl. 15, 439 (1971).

${ }^{3}$ V. Zakharov, V. L'vov, and G. Falkovich, Kolmogorov Spectra of Turbulence (Springer, Berlin, 1992).

${ }^{4}$ E. A. Kuznetsov, Zh. Eksp. Teor. Fiz. 120, 1213 (2001) [ JETP 93, 1052 (2001)].

${ }^{5}$ In the case of three-wave interacting waves of different branches, the criterion of weak turbulence consists in the smallness of the inverse nonlinear time defining by the kinetic equations in comparison with the maximal growth rate of the corresponding decay instability for the monochromatic wave. The latter in such system represents the inverse time of randomization (see, e.g., Ref. 4).

${ }^{6}$ V. I. Arnold, Catastrophe Theory (Znanie, Moscow, 1981; Springer, Berlin, 1986); Mathematical Methods of Classical Mechanics, 3rd ed. (Nauka, Moscow, 1984; Springer, New York, 1989).

${ }^{7}$ V. V. Krasnoselskikh, Sov. Phys. JETP 62, 282 (1985).

${ }^{8}$ S. F. Shandarin and Ya. B. Zeldovich, Rev. Mod. Phys. 61, 185 (1989).

${ }^{9}$ L. D. Landau and E. M. Lifshitz, The Classical Theory of Fields (Pergamon/Addison-Wesley, London/Reading MA, 1959).

${ }^{10}$ G. B. Witham, Linear and Nonlinear Waves (Wiley Interscience, New York, 1974).

${ }^{11}$ A. V. Gurevich and L. P. Pitaevskii, in Reviews of Plasma Physics, edited by M. A. Leontovich (Consultants Bureau, New York, 1986), Vol. 10, p. 1.

${ }^{12}$ O. M. Phillips, J. Fluid Mech. 4, 426 (1958).

${ }^{13}$ P. G. Saffman, Stud. Appl. Math. 50, 49 (1971).

${ }^{14}$ E. A. Kuznetsov, Pis'ma Zh. Eksp. Teor. Fiz. 80, 92 (2004) [JETP Lett. 80, 83 (2004)].

${ }^{15}$ E. A. Kuznetsov, V. Naulin, A. H. Nielsen, and J. J. Rasmussen, Phys. Fluids 19, 105110 (2007).

${ }^{16}$ B. B. Kadomtsev and V. I. Petviashvili, Dokl. Akad. Nauk SSSR 208, 794 (1973) [Sov. Phys. Dokl. 18, 115 (1973)].

${ }^{17}$ V. S. L'vov and G. E. Falkovich, Sov. Phys. JETP 53, 299 (1981).

${ }^{18}$ V. S. L'vov, Yu. L'vov, A. C. Newell, and V. Zakharov, Phys. Rev. E 56, 390 (1997)

${ }^{19}$ E. A. Kuznetsov, Zh. Eksp. Teor. Fiz. 62, 584 (1972) [Sov. Phys. JETP 35, 310 (1972)].

${ }^{20}$ R. S. Iroshnikov, Astron. Zh. 40, 742 (1963) [Sov. Astron. 7, 566 (1964)]; R. H. Kraichnan, Phys. Fluids 11, 945 (1965).

${ }^{21}$ S. Galtier, S. V. Nazarenko, A. C. Newell, and A. Pouquet, J. Plasma Phys. 63, 447 (2000); S. V. Nazarenko, A. C. Newell, and S. Galtier, Physica D 152-153, 646 (2001).

${ }^{22}$ G. Falkovich and M. Meyer, Phys. Rev. E 54, 4431 (1996). 